\title{
Clinical introduction into population health management using a peer mentoring strategy
}

\author{
Margaret A. Avallone,, Renee Cantwell, Staci Pacetti \\ School of Nursing-Camden, Rutgers University, Camden, New Jersey, United States
}

Received: October 21, 2018

Accepted: December 18, 2018

Online Published: December 24, 2018

DOI: $10.5430 /$ jnep.v9n4p79

URL: https://doi.org/10.5430/jnep.v9n4p79

\begin{abstract}
Background/objective: Baccalaureate nursing clinical experiences must prepare graduates to assess and support the health of vulnerable populations within communities. Clinical experiences need to align with theoretical coursework throughout the entire curriculum. This pilot project evaluated an innovative method to introduce second-semester Accelerated Baccalaureate Students (ABS) into a service-learning community experience using a peer-mentoring strategy.

Methods: Eleven second-semester and twelve fourth-semester ABS students were paired in learning dyads in a low-income, ethnically diverse urban housing development, along with community health workers and social workers. Second-semester students were peer-mentored by fourth-semester students. Second-semester students performed health screenings, health promotion education, and medication reconciliation guided by fourth-semester students. Learning objectives and changes in knowledge were evaluated before and after the experience in a retrospective pretest/posttest format for all students. Additionally, second-semester students reported their perception of the mentoring experience on self-confidence, satisfaction, and helpfulness.

Results: The learning objective rated highest related to the role of social determinants of health in the overall health of the residents $(\mathrm{M}=4.38)$. Paired $t$-test analysis revealed significant positive increases in levels of knowledge about social determinants of health, role of culture, and importance of the interprofessional team. On a scale of one to five, second-semester students reported increased self-confidence $(M=4.2)$, satisfaction with the learning experience $(M=4)$, and help providing health promotion strategies $(\mathrm{M}=4.4)$ due to mentorship by the fourth semester students.

Conclusions: Students' comments reflected an appreciation of the complexity of healthcare issues affecting vulnerable members of the community. Second-semester students reported more confidence and perceived themselves to be more effective due to the mentorship of the fourth semester students when providing education to residents with complex health needs.
\end{abstract}

Key Words: Service-learning, Baccalaurate nursing community clinical, Peer mentorship

\section{INTRODUCTION}

Education for future healthcare professionals requires a curriculum that shifts its focus from an acute illness to a prevention and population health management framework. ${ }^{[1]}$ A nursing curriculum with an acute illness context fails to clearly illuminate the social determinants of health which, in some populations, can lead to the development of chronic diseases as well as the inability to manage these diseases. Furthermore, providing predominantly clinical experiences in acute care settings does not adequately prepare the future graduate for practice in the 21 st century where more and more healthcare will focus on health promotion and disease prevention in the community. ${ }^{[2]}$ Clinical education and experiential learning must align with the theoretical course

\footnotetext{
* Correspondence: Margaret A. Avallone; Email: margaret.avallone@ rutgers.edu; Address: School of Nursing-Camden, Rutgers University 530 Federal St., Camden NJ, United States.
} 
work in community and population health. The Institute of Medicine has prioritized the enhancement of nursing clinical education in community settings and population health managment. ${ }^{[3]}$

In one accelerated nursing program, a curriculum revision was the impetus for introducing clinical experiences aimed at increasing student competencies in population health. This article will describe the pilot of a unique undergraduate service-learning experience where second-semester accelerated baccalaureate nursing students $(\mathrm{ABS})$ engaged socially and economically disadvantaged, primarily Spanishspeaking geriatric residents in a low-income, urban highrise residential setting. This early experience in community health was accomplished by pairing the second-semester students with fourth-semester students using an innovative peer mentorship strategy. The article will describe early pilot project experiences as well as next steps.

\subsection{Integration of population health management clini- cal experiences}

Nursing graduates must have competencies to meet the complex healthcare needs of an aging, diverse and vulnerable population. By 2060, 20 million Americans will be 85 years of age or older. Twenty-two percent of the population 65 years and older will be Hispanic, 55 percent non-Hispanic White, 12 percent non-Hispanic Black, and 9 percent nonHispanic Asian. ${ }^{[4]}$

The AACN Baccalaureate Essentials identify the need for graduates to assist older individuals in the management of common geriatric syndromes. ${ }^{[5]}$ Nursing programs must incorporate population-focused didactic content as well as clinical learning opportunities into the baccalaureate nursing curriculum. In particular, clinical experiences in community agencies and senior centers can assist students in learning about common geriatric syndromes, assessment of older adults' efficacy in self-care, and ability to manage chronic health conditions. ${ }^{[6]}$ In this way, nursing schools can provide opportunities to learn essential skills that baccalaureate nurses will need to promote population health. ${ }^{[1]}$

In one accelerated nursing program, community experiences in the final semester provided an opportunity for nursing students to apply knowledge and skills acquired in the first three semesters. These particular community experiences only partially fulfilled the acquisition of the knowledge, skills, and attitudes needed to practice in the 21 st Century. Faculty were concerned that, by not addressing population health management concepts including social determinants of health until the final semester, student professional identity and attitudes regarding the poor, the aged, and the disadvantaged might be largely formed. A revision of the ABS curriculum was undertaken in order to more fully address nursing students' education relating to population health. In the curriculum revision, the original Adult Health course for second-semester nursing students was revised to address population health management concepts and retitled "Health and Illness in Adult Populations". The corresponding clinical course was also revised accordingly. By providing early clinical experiences in a community setting in addition to the clinical experiences in acute care, it was hoped that student nurses would develop a greater understanding and appreciation of the barriers that underserved populations face when managing chronic diseases in the community. Furthermore, a formal experience with a marginalized elder population might positively influence student attitudes. ${ }^{[7]}$

\subsection{Service-learning in nursing}

Service-learning is one pedagogy used to provide meaningful community services for marginalized populations while meeting learning goals. ${ }^{[8]}$ Service-learning links educational objectives with service intended to benefit the community, and has been demonstrated to heighten a sense of civic responsibilities and social justice. ${ }^{[9,10]}$ By introducing students to nursing care of residents in a disadvantaged community setting, it was hoped that students would gain a first-hand appreciation of the role that social determinants play on the health of a population.

\subsection{Peer mentoring}

Peer mentoring is defined as a supportive relationship between a novice and a more experienced person which exists to meet the developmental needs of the novice. ${ }^{[11]}$ Peer mentorship has been used successfully in a number of settings, including nursing education, to enhance cognitive and psychomotor development, increase confidence and satisfaction with the learning experience, and promote professional development. ${ }^{[12,13]}$ This method of learning also supports concepts of teamwork and develops leadership and communication skills which are important for providing quality nursing care. ${ }^{[13]}$ Peer mentorship is consistent with Knowles' Adult Learning Theory, which supports the use of mentors, peers, and others, to meet the varied needs of the adult learner. ${ }^{[14]}$

Novice nursing students with limited clinical experience can be anxious and have lower self-confidence in their knowledge base and skill set. Peer teaching and learning and peer mentorship in the clinical setting has been shown to increase student confidence, reduce anxiety, improve learning, reduce feelings of vulnerability, and assist communication skills. ${ }^{[13,15,16]}$ The partnership of fourth-semester students and second-semester students was used effectively to teach 
heart failure transitional education in the classroom . Avallone and Cantwell found that upper level nursing students were able to relate in a positive way to the lower level nursing students, resulting in decreased levels of anxiety and higher self-esteem and self-confidence for lower level students. ${ }^{[17]}$

\section{DESCRIPTION OF PROJECT}

The planning team designed a student peer mentorship framework to facilitate a health promotion and disease management community service-learning experience in order to satisfy the Health and Illness in Adult Populations course clinical objectives. The service-learning experience was conducted in an urban subsidized high-rise development that provided homes to elderly, economically and racially diverse residents. A partnership was developed between the School of Nursing and the Social Service department in the high-rise development, and a memorandum of agreement was signed. The Social Service department had an established social service and wellness program for residents, and utilized community health workers as well as social workers, students and pastoral ministry volunteers to meet health needs of the building residents.

Second-semester ABS nursing students who were enrolled in the newly revised Health and Illness in Adult Populations course, and fourth-semester students who were enrolled in the Community Health course were eligible to participate in the service-learning project. Students were able to volunteer for the project, but not every student in each course was able to participate. Interprofessional teamwork and peer mentorship are concepts discussed in the fourth semester Leadership course. These concepts were enhanced by grouping a secondsemester nursing student and a fourth-semester student with a member of the social service team. Fourth-semester students were utilized as peer mentors for the second-semester students. Second-semester nursing students were not yet prepared to independently provide health promotion and disease prevention interventions, skills needed to care for the residents of the housing development. Second semester students began the semester with minimal chronic disease management knowledge and no community health experience, while the fourth semester students came to the experience with two semesters of medical-surgical nursing, pharmacology, one semester each of maternal health, pediatrics, and mental health and were currently enrolled in Leadership and Community Health nursing courses. The Social Service team member, as the third member of the group, identified residents to interview and assess, provided resident background information, and assisted in follow-up of care issues and referrals when students could not accomplish these things during the clinical experience. The Social Service team proPublished by Sciedu Press vided translation services when necessary as well.

The clinical course competencies were derived from the AACN Baccalaureate Essential VII: Clinical Prevention and Population Health. ${ }^{[6]}$ By the end of the service-learning project, both second and fourth semester students were expected to meet the following learning objectives:

- Identify social determinants of health impacting resident health outcomes.

- Explore the link between culture and its influence on individuals, communities and provision of culturally appropriate and sensitive health care.

- Use evidence-based strategies to guide health teaching, health counseling, and screening in a culturallydiverse, socially disadvantaged geriatric population.

- Collaborate with other health professionals and residents to meet individual and family-centered needs within the context of the interprofessional team to identify and utilize available and appropriate communitybased services for the assigned resident. ${ }^{[6]}$

To meet these competencies, the Social Service team identified residents who consented verbally to health visits from nursing students. Residents targeted for visits included those recently discharged from an inpatient facility, residents with two or more chronic diseases or a history of multiple hospitalizations who may benefit from chronic disease selfmanagement education. The Social Service team also identified residents with potential safety or fall risk concerns.

The clinical experience was offered over a course of a 15week semester. Participating students rotated out of acute care clinical experiences for two four-hour days each week, allowing for two different students weekly. The Social Service team members were consistent since they were members of the staff. On the first four-hour day, the team would visit two to three identified residents. During each resident visit, the nursing students performed a health needs assessment, identified resident health concerns, and began health education and medication reconciliation. Students were supported by a PharmD faculty member and Advanced Practice Nurse (APN) faculty members who were on site and available for consulation. On the second four-hour day, students followed up on any issues or problems identified from the first visit with residents. At the end of day two, an interprofessional team-based case presentation was held. The interprofessional case presentations led by the second-semester student and mentored by the fourth semester student promoted team work and communication. Problems were identified and potential solutions were generated.

At the completion of the experience, all second and fourthsemester students were asked to complete a clinical evalua- 
tion survey as well as a survey evaluating change in knowledge. Furthermore, second-semester students were asked to complete a questionaire evaluating their experience working with the fourth-semester peer mentor. The survey questionaires were submitted anonymously. No HIPAA identifying information was collected (see Tables 1-3).

Table 1. Learning objectives

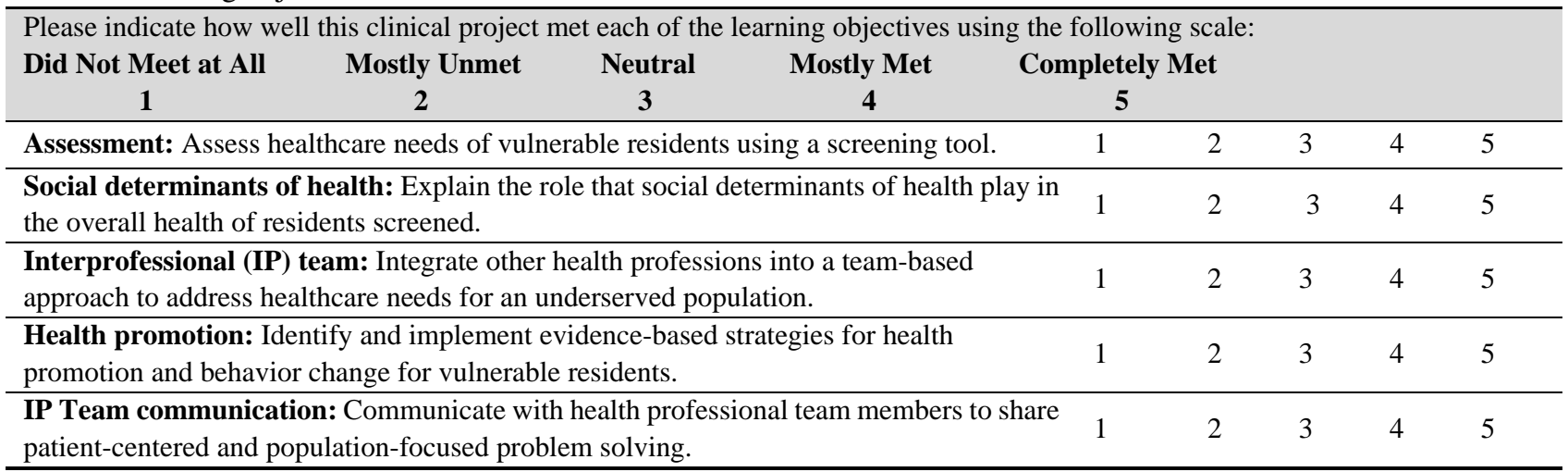

Table 2. Change in knowledge level

\begin{tabular}{|c|c|c|c|c|c|c|c|c|c|c|}
\hline \multirow{2}{*}{\multicolumn{6}{|c|}{$\begin{array}{l}\text { Please circle the number that represents how you rate your know } \\
\text { 1-novice 2-advanced beginner } 3 \text { competent } 4 \text { proficient } 5 \text { expert } \\
\text { Knowledge before experience }\end{array}$}} & \multirow{2}{*}{\multicolumn{5}{|c|}{ Knowledge after experience }} \\
\hline & & & & & & & & & & \\
\hline 1 & 2 & 3 & 4 & 5 & $\begin{array}{l}\text { Explain the link between culture and its influence on individuals, } \\
\text { communities, and the provision of culturally-appropriate health care. }\end{array}$ & 1 & 2 & 3 & 4 & 5 \\
\hline 1 & 2 & 3 & 4 & 5 & $\begin{array}{l}\text { Explain the relationship between social determinants of health, health } \\
\text { risk factors, and client outcomes. }\end{array}$ & 1 & 2 & 3 & 4 & 5 \\
\hline 1 & 2 & 3 & 4 & 5 & $\begin{array}{l}\text { Describe opportunities for advocacy and collaboration with community } \\
\text { and clinical partners that can be used to address unmet client needs. }\end{array}$ & 1 & 2 & 3 & 4 & 5 \\
\hline 1 & 2 & 3 & 4 & 5 & $\begin{array}{l}\text { Identify the importance of integrating client and community values and } \\
\text { priorities/preferences into health care decisions. }\end{array}$ & 1 & 2 & 3 & 4 & 5 \\
\hline 1 & 2 & 3 & 4 & 5 & $\begin{array}{l}\text { Identify the importance of an interprofessional team-based approach } \\
\text { when making healthcare decisions. }\end{array}$ & 1 & 2 & 3 & 4 & 5 \\
\hline
\end{tabular}

Additionally, all students were asked to reflect on the experience by answering several open-ended questions.

1) What is the most important thing you learned from this experience?

2) Provide one example of how you plan to utilize the information you learned during this experience?

3) What did you like best about this experience?

4) What would you change or improve about this experience?

Reflections were used for two purposes. First, reflection is an integral component of the service-learning pedagogy. ${ }^{[18]}$ Also, as a pilot experience, the investigators utilized the student reflections to identify opportunities to improve the experience.

\section{RESUlts}

A total of 21 nursing students participated in the population health managment peer mentorship pilot program, 10 of whom were second-semester students and 11 were fourthsemester students Students evaluated how well the program met each learning objective (on a 5-point scale from "Did not meet at all" to "Completely Met"). The highest rating provided was for the learning objective related to explaining the role of social determinants of health in the overall health of the residents screened $(\mathrm{M}=4.38)$. The mean rating for all other learning objectives fell between "Neutral" and "Mostly Met" (assess health care needs $\mathrm{M}=3.86$, health promotion $\mathrm{M}=3.71$, IP team communication $\mathrm{M}=3.43$, and IP team approach $\mathrm{M}=3.33$ ).

In addition, all students assessed their knowledge level in five content areas before and after the experience in a retrospective pretest/posttest format (on a 5-point scale from Novice to Expert). Paired $t$-test analyses indicated that there were significant positive increases in the level of knowledge reported (average of nearly a 1-point increase, 0.86 ) for all five content areas (culture and health care, social determinants of 
health, advocacy and collaboration, integrating patient values in health care decisions, importance of interprofessional team-based approach).

Nine second-semester students (out of 10) completed the survey evaluating their experience working with the fourth semester peer mentor. Students rated the effectiveness of the peer mentors on a 5-point scale from Strongly Disagree to Strongly Agree (see Table 3).

Table 3. Peer mentor evaluation

\begin{tabular}{ll}
\hline Effectiveness of Peer Mentors: & Mean (M) \\
\hline Helped me understand needs of vulnerable populations & 3.6 \\
Helped me understand social determinants of health & 3.2 \\
Made the learning experience more satisfying & 4.4 \\
Helped me implement health promotion interventions & 4.0 \\
Increased my confidence level & 4.2 \\
\hline
\end{tabular}

Within their reflections, students provided valuable feedback regarding their experience. Overall, students indicated that it was a very worthwhile experience with many identifying specific plans for utilizing the information learned. Several students noted how the social determinants of health really impact health and that "seeing it made it much more real". They relayed an understanding of how an individual's home, culture, language and environment may affect their health management plans.

Students reflected on the importance of safe transition of care upon discharge from an acute care facility. "Most residents don't understand the significance of their hospitalization, what happened during their stay, and how to manage their condition to prevent a readmission". They identified how important it was for discharged patients to "close the loop" once back in the home. Using the information learned, the students stated that they will view patients in a more holistic way in the future and "assess the social and environmental situation, transportation, and financial resources and incorporate these into discharge planning".

When asked what they liked best, the students particularly enjoyed the "exposure to a completely different side of healthcare" by meeting people where they reside rather than in a hospital room. They identified an appreciation for the insight they gained about the residents as "individuals" and how this insight helped them develop a more "patient-centered perspective" when providing health education.

Second-semester students also stated that they enjoyed the partnership they developed with the fourth-semester nursing students, recognizing the value of peer mentorship in education. One student commented that "it was an unfamiliar setting for me. Having the fourth-semester student made the experience much nicer and more relaxing. I felt calmer and more confident having a more senior student there as a back-up". Another student stated, "I did not know what or how to teach about managing diseases at home, but the fourth-semester student gave me great ideas. It gave me hope that I will have this knowledge in a few semesters". Another student, a dietitican prior to returning for nursing school stated, "we were able to learn from each other. She taught me things and I was able to teach her things that I knew from my work as a dietitian".

Finally, the students provided constructive feedback regarding methods for project improvement. The overarching theme was to "spend more time with the residents consistently in order to make meaningful connections, establish a presence, and build trust". In addition, prior to the first resident visit, the students suggested that they have access to more background information about the resident in order to be better prepared to engage and ask appropriate questions.

\section{Discussion}

Overall, both second and fourth-semester students evaluated the experience positively and met the curricular objectives. Students reported an increase in knowledge in all five content areas assessed. Though the entire group together (both second and fourth-semester students) reported an improvement in knowledge as a result of the experience, the small number of participants in each of the groups precluded an evaluation regarding the effect of the peer mentorship relationship on change in knowledge. This was a feasibility pilot and the purpose was not to ascertain the effect of peer mentorship on knowledge. Larger sample sizes will be required to determine significant effect in future studies.

Students appreciated the opportunity to assess health needs and provide health education in the resident's home. The most common criticism was that the experience was too short. In order to accommodate the peer mentoring aspect of this project, second-semester students rotated out of acute care medical-surgical experiences for one week. Fourth-semester students rotated out of a community experience. While the two-day clinical experience was perceived as valuable to students, the majority recommended adding substantial time in the rotation to facilitate therapeutic relationships with the residents. To assist all students in relationship building and interviewing techniques, future semesters will be structured with opportunities to re-visit residents over seven weeks rather than two weeks.

Moving from an acute care focus to a community and population health managment focus, second-semester students at times, lacked adequate interviewing and communication 
skills to establish a therapeutic relationship. In some cases, these students seemed focused on filling out the assessment forms and missed important verbal or body language cues from the resident. To help second-semester students build necessary communication skills, scripting, role-play and lowfidelity simulation with their peer mentor will be employed as part of the orientation to the experience.

According to the nine second-semester students who completed the peer mentor questionnaire, the mentorship by the fourth-semester students increased their confidence, made the learning experience more enjoyable, and facilitated their ability to provide health promotion interventions. Though this survey was limited due to the low number of mentees, the preliminary findings suggest that the second-semester students found the peer mentorship beneficial. In a scoping review, Steven Jacobs found that mentees felt supported and less vulnerable working together. ${ }^{[16]}$ Also, the mentoring relationship has been found to assist participant communication skills. ${ }^{[16]}$ Additional studies suggest that peer mentorship in the clinical setting reduces anxiety, increases confidence, and improves learning. ${ }^{[13,15]}$ In this community setting, the second-semester students came to the experience with minimal clinical knowledge and experience from which to draw. It is surmised that the peer mentors provided a sense of security and support to enable learning to occur. Though most second-semester students valued the peer mentorship relationship, others scored the helpfulness and impact of the mentorship experience as "neutral". In future semesters, peer mentors will be better educated and prepared for their role prior to the start of the experience.

It is unknown if the peer mentor students benefitted from the experience, as the fourth-semester students were not surveyed about their experience as Peer Mentors, an acknowledged limitation of this pilot. Studies have suggested that peer mentors benefit from the interaction as well as the mentee. ${ }^{[11,16]}$ Explaining new concepts to other learners may enhance learning and facilitate intergration of previously learned concepts. ${ }^{[11]}$ Future semesters will survey the peer mentors as well as the mentees to better understand the effect of the experience.

Additional opportunities to improve the clinical experience were identified during the implementation of the pilot project. Occasionally, no baseline information was available prior to students' interviewing the residents, making needs assessment difficult. Students also indicated a need to have printed patient education available immediately in both English and Spanish. Finally, communication barriers due to the high number of Spanish speaking residents made it sometimes difficult to assess the resident and provide appropriate inter- vention.

About $50 \%$ of the building residents were Spanish speaking, yet none of the students spoke Spanish. Though students were accompanied by a Spanish speaking community healthcare worker during each visit, the language difference imposed a barrier when developing a therapeutic and trusting relationship. To build future capacity caring for Spanishspeaking clients, the School of Nursing is promoting the "Spanish for Health Professionals" elective course for all nursing students. Additionally, Spanish-speaking students will be identified and asked to pair with a non Spanishspeaking student.

\subsection{Moving forward}

This article describes the pilot of a service-learning project designed to place nursing students into community settings earlier in the curriculum using a peer mentoring strategy. Second-semester students partnered with fourth-semester students to perform health promotion, disease management education, and medication reconcilitation and medication education in a geriatric low-income housing setting. The purpose of the pilot was to assess the feasibility of scheduling a peer mentoring community clinical experience with second and fourth-semester students partnered together. The pilot helped the planning team identify early successes and opportunities for improvement. In subsequent semesters, the planning team will focus on specific health outcomes for the elderly population within the housing development, including fall prevention, dementia screening, and reduction of medication-related adverse events. Additionally, the team will incorporate additional education and role training for the Peer Mentors prior to the clinical experience. The effects of the peer mentorship relationship on both mentor and mentee will be studied. Additionally, the team plans to measure the effects of an early service-learning experience on civic and social responsibility in second semester nursing students and evaluate students' ability to work as interprofessional team members.

Because the residential building cannot accommodate an entire class of nursing students, the rotation will continue to include those who volunteer. However, efforts will be made to offer a similar experience for all students in other residential facilities. This will require extensive community outreach and collaboration. Each residential building is unique and all may not provide the extent of social services that is offered in this facilty.

Nursing care is not only provided in acute care settings; more and more nursing care is provided in the community to various populations. Students need to participate early and often 
in service learning experiences during their nursing program. Innovative strategies must be employed to support novice learners in unfamiliar settings. Peer mentoring may be one such strategy that may show promise. This strategy needs further investigation. By moving nursing students into the

\section{REFERENCES}

[1] AACN. Public Health: Recommended Baccalaureate Competencies and Curricular Guidelines for Public Health Nursing: A Supplement to the Essentials of Baccalaureate Professional Nursing Practice. September, 2013.

[2] Institute of Medicine (IOM), For the Public's Health: Investing in a Healthier Future., Washington, D.C.: National Academies Press, 2012. Available from: http://www.nationalacademies.org/ hmd/Reports/2012/For-the-Publics-Health-Investing -in-a-Healthier-Future.aspx

[3] Institute of Medicine (IOM). The Future of Nursing: Leading Change, Advnancing Health. Washington, D.C.: national Academies Press; 2010. Available from: https://www.nap.edu

[4] Federal Interagency Forum on Aging-Related Statistics Older Americans 2016: Key indicatiors of well-being. Federal Interagency Forum on Aging-Related Statistics. 2016. Washington, D.C.: U.S. Government Printing Office.

[5] Association of Community Health Nursing Educators (ACHNE). Essentials of Baccalaureate Nursing Education for Entry Level Community/Public Health Nursing. Wheat Ridge, CO, 2009. Available from: http://achne.org/files/Essentials0f $\backslash T 1 \backslash t e$ xtquotedblrightBaccalaureate_Fall_2009.pdf

[6] AACN. The Essentials of Baccalaureate Education for professional nursing practice. Available from: http://www. aacn.nche.edu/ education-resources/BaccEssentials08.pdf

[7] Teal CM, Gill A, Green A, et al. Helping medical learners recognize and manage unconscious bias toward certain patient groups. Med Education. 2012; 46: 80-88. PMid:22150199 https://doi.org/ $10.1111 / j .1365-2923.2011 .04101 . x$

[8] Jarrell K, Ozymy J, Gallagher J, et al. Constructing the foundations for compassionate care: How service-learning affects nursing students' attitudes towards the poor. Nurse Education in Practice. 2014; 14: 299-303.

[9] Groh CJ, Stallwood, Daniels JJ. Service-learning in nursing education: Its impact on leadership and social justice. Nursing Education community earlier and with more purpose, nursing schools can graduate nurses who are able to meet the nursing care demands of the future.

\section{CONFLicts of InTEREST Disclosure}

The authors declare that there is no conflict of interest.
Perspectives. 2011; 32(6): 400-5. https://doi.org/10.5480/15 36-5026-32.6.400

[10] Nokes KM, Nickitas DM, Keida R, et al. Does service-learning increase cultural competency, critical thinking, and civic engagement? Journal of Nursing Education. 2005; 44(2): 65-70.

[11] Bene KL, Bergus G. When learners become teachers: A review of peer teaching in medical student education. Family Medicine. 2014; 46(10): 783-7. PMid:25646829

[12] Goldsmith M, Stewart L, Ferguson L. Peer learning partnership: an innovative strategy to enhance skill acquisition in nursing students. Nursing Education Today. 2006; 26: 123-130. PMid:16202483 https://doi.org/10.1016/j.nedt.2005.08.001

[13] Secomb J. A systematic review of peer teaching and learning in clinical education. Journal of Clinical Nursing. 2007; 17: 703-716. PMid:18047577 https://doi .org/10.1111/j.1365-2702.20 $07.01954 . \mathrm{x}$

[14] Knowles M. The Adult Learner: A Neglected Species, third ed. Houston: Gulf Publishing Co.; 1984.

[15] Austria MJ, Baraki K, Doig AK. Collaborative learning using nursing student dyads in the clinical setting. International Journal of Nursing Education Scholarship. 2013; 10(1): 73-80.

[16] Jacobs S. A scoping review examining nursing student peer mentoring. Journal of Professional Nursing. 2017; 33: 212-223. PMid:28577814 https://doi.org/10.1016/j.profnurs. 201 6.09 .004

[17] Avallone MA, Cantwell R. Teaching nursing students to provide effective heart failure patient education using a peer teaching strategy. Journal of Nursing Education and Practice. 2017; 7(2): 84-89. https://doi.org/10.5430/jnep.v7n2p84

[18] Schmidt NA, Brown JM. Service learning in undergraduate nursing education: Strategies to facilitate meaningful reflection. Journal of Professional Nursing. 2016; 32(2): 100-106. PMid:27000194 https ://doi.org/10.1016/j.profnurs . 2015.06.006 